\section{Customized Television: Standards Compliant Advanced Digital Television}

Michel Bais, John Cosmas, Christoph Dosch, Andreas Engelsberg, Alexander Erk, Per Steinar Hansen, Pat Healey, Gunn Kristin Klungsoeyr, Ronald Mies, Jens-Rainer Ohm, Yakup Paker, Alan Pearmain, Lena Pedersen, Åsmund Sandvand, Rainer Schäfer, Peter Schoonjans, and Peter Stammnitz

\begin{abstract}
This correspondence describes a European Union supported collaborative project called CustomTV based on the premise that future TV sets will provide all sorts of multimedia information and interactivity, as well as manage all such services according to each user's or group of user's preferences/profiles. We have demonstrated the potential of recent standards (MPEG-4 and MPEG-7) to implement such a scenario by building the following services: an advanced EPG, Weather Forecasting, and Stock Exchange/Flight Information.
\end{abstract}

Index Terms-Digital TV, image coding, interactive TV, TV equipment.

\section{INTRODUCTION}

The introduction of digital television offers exciting possibilities for new services by adding multimedia enhancements to broadcasts. It is important that the consumer should see digital television as not just an increased choice of programs, but as a new way of accessing services and information. Current digital broadcast systems already provide some means of navigation through channels and added value services. However, these often rely on proprietary systems, such as OpenTV. The European Broadcasting Union Digital Video Broadcasting Project (DVB) has only recently started to put greater effort into establishing the Multimedia Home Platform (MHP) standard for added value services [1], [2]. Normal DVB broadcasts use the MPEG-2 standard developed by the Motion Picture Experts Group. However, the subsequent standards developed by the group, namely MPEG-4 and MPEG-7 have yet to be used by broadcasters for novel services either in digital broadcasting or for material delivered via the Internet. The object-oriented multimedia coding standard MPEG-4 enables efficient coding of several multimedia streams and compositing these in a scene. The scene could include several video objects, transmitted as separate streams, and placed at different locations according to the scene description. The objects could be of different sizes and transmitted at different bit rates. MPEG-4 also provides a means of user interaction with the scene [3]. The MPEG-7 standard provides a

Manuscript received July 13, 2001; revised March 18, 2002. This work was supported by the European Union Advanced Communication Technologies and Services Programme, Project AC 360.

M. Bais and R. Mies are with KPN Research, Leidschendam NL-2260 AK, Netherlands (e-mail: R.J.M.Mies@kpn.com).

J. Cosmas is with Brunel University, Uxbridge, Middlesex UB8 3PH, England (e-mail: john.cosmas@brunel.ac.uk).

C. Dosch, A. Erk, and R. Schäfer are with the Institut für Rundfunktechnik GmbH, Munich D-80939, Germany (e-mail: dosch@irt.de).

A. Engelsberg is with Robert Bosch GmbH, Hildesheim D-31139, Germany. P. S. Hansen, G. K. Klungsoeyr, L. Pedersen, and Å. Sandvand are with Telenor N-2027 Kjeller, Norway (e-mail: gunn-kristin.klungsoyr@telenor.com).

P. Healy, Y. Paker, A. Pearmain, and P. Schoonjans are with Queen Mary, University of London, London E1 4NS, England (e-mail: alan.pearmain@elec.qmul.ac.uk).

J.-R. Ohm and P. Stammnitz are with Heinrich-Hertz-Institut fuer Nachrichtentechnik Berlin GmbH, Berlin D-10587, Germany (e-mail: stammnitz@hhi.de).

Publisher Item Identifier S 0018-9316(02)05256-3. framework for content indexing and allows metadata to be added to multimedia information [4]

CustomTV was a European Union-supported collaborative project funded under the ACTS program that finished at the end of 1999. CustomTV was based on the premise that future TV sets will have to provide the functionality to select and present information and services according to each user's preferences/profiles. The objective of CustomTV has been to utilize the existing digital broadcast technology for transmitting all forms of multimedia information on top of normal digital TV channels without necessarily requiring a return channel. All streams are associated with metadata descriptors that could then be used, at each receiver, to customize the information received. The screen display can be organized according to viewer preferences established by an intuitive user interface and prerecorded user profiles.

CustomTV has attempted to implement what could be called "objectbased interactivity" and "program and subprogram selection." These capabilities have been implemented by relying on the MPEG-4 and MPEG-7 standards with the expectation that this will increase interest in the standards within the broadcasting and the Internet world and lead to a closer link between digital television and Internet applications.

The customized TV system that has been designed uses the meta-data descriptors that are associated with the $\mathrm{A} / \mathrm{V}$ information streams received to customize the information presented to the receiver screen. The way the screen is organized for the display is achieved according to viewer preferences established by user profiles. For example, if the user's preferences have been established to be sports programs then these programs are presented to the viewer. User preferences may be associated with an event at the program level, such as the start of a football sports program, but it may also be associated with an event at the subprogram level, such as the scoring of a goal within a football sports program. The customized TV system allows users to define, through the definition of user preferences, events at both the program level and at the subprogram level.

The customized TV system also allows additional information to be accessed by the selection of objects that launch additional text, audio, or video information. For example, the selection of objects within a film or program may launch some advertising related to a program. Selection of the listing of company stock prices could launch an exchange ticker giving variations of prices. Tools have been developed for media content providers to define events and objects using standards-compliant methods to assist selection at the consumer end.

A working demonstrator with a customized and interactive display screen was developed that receives broadcast MPEG-2 and MPEG-4 content with MPEG-7 indexing, via satellite or other DVB networks. MPEG-2 and MPEG-4 video can be displayed and arranged within the customized display screen. Areas within the MPEG-4 video can be selected to launch further 2-D MPEG-4 graphics or video to present additional information.

\section{Customized TV Service Scenarios}

In order to focus our work, a number of representative scenarios were selected.

\section{A. Advanced Electronic Program Guide (EPG) Using MPEG-4}

This version of Electronic Program Guide (EPG) offers two enhancements over those currently available. MPEG-4 is used to provide trailers of current programs, shown four at a time on the screen, one of which can then be selected via the user interface. The second enhancement is that the trailers displayed are selected from all 


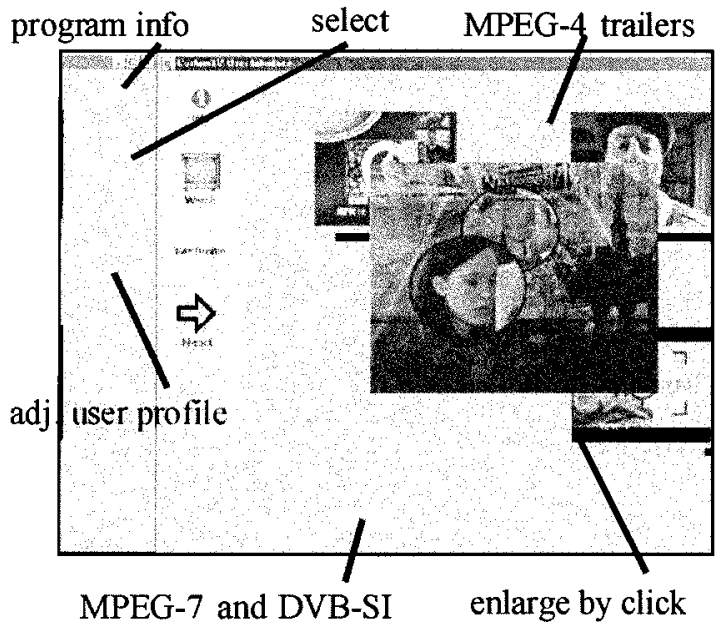

Fig. 1. EPG.

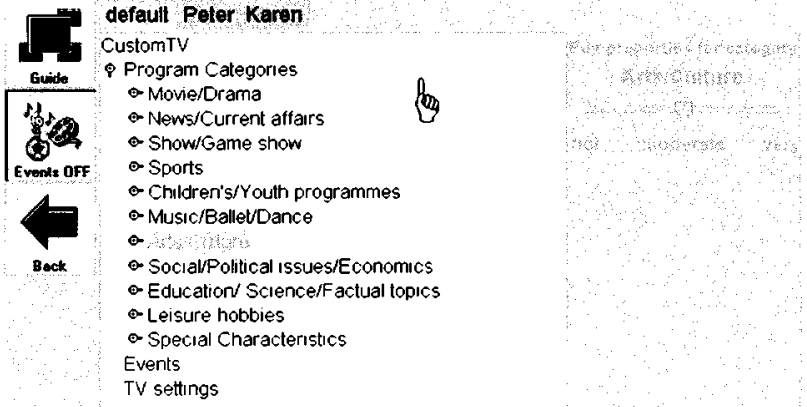

Hurricane is threatening 10 make millions of

homeless people in Miami!

Fig. 2. Adjustment of program preferences.

the available programs according to the stored preferences of the user, using MPEG-7 metadata added to the programs. The resulting EPG is shown in Fig. 1.

The material for TV programs was divided into the categories of news, sports, film/movie, cartoons/kids, talk shows/daily interest, etc. The channels had MPEG-7 data associated with the MPEG-2 program content and this was displayed in the yellow information area at the bottom of the EPG. If full information on the content was required then selecting Program Info provided all the MPEG-7 information that was associated with the particular program content. The user could also choose to adjust or redefine his/her private profile by selecting Adj. User Profile, which launches the screen shown in Fig. 2.

In the user profile setup, the user is presented with the set of categories, level1 and level2, arranged in the shape of a tree. Using the buttons on a remote control, the user is able to traverse the tree. When a new category or subcategory is selected, its properties are fetched from the user's profile and displayed at the bottom of the screen. The viewer can then specify his/her interest at category and subcategory levels. The EPG can generate a personal profile for more than one user, or one user can have more than one profile.

The EPG allows the viewer to be notified of content on any other channels that have a high interest rating in his/her personal profile.

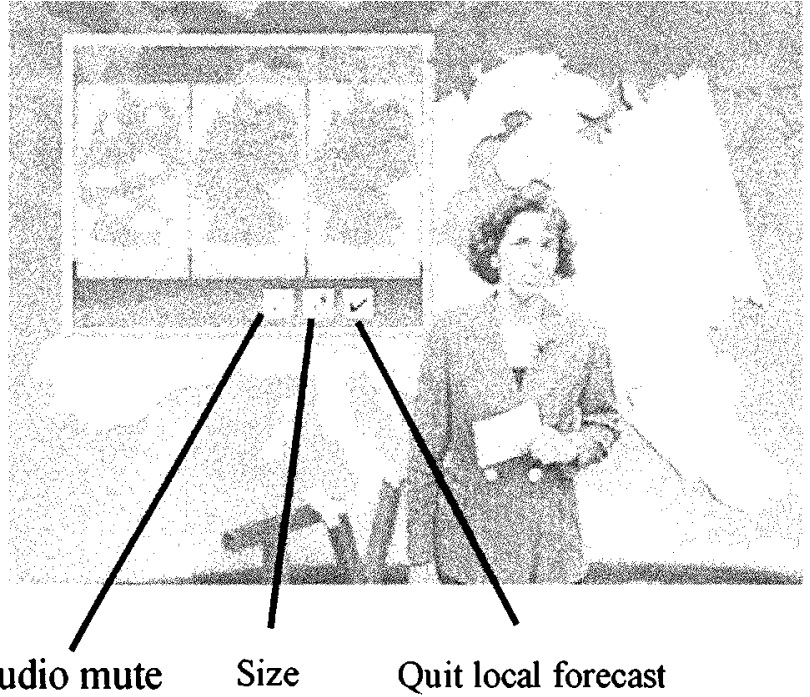

Fig. 3. Weather scenario.

An example of this is a viewer being notified when a football goal (a subprogram event) is scored on another channel, while watching a film. The occurrence of this event launches a select icon that allows the viewer to view the channel on which the replay of the goal is shown. A similar situation is the launching of a select icon to notify the viewer of an emergency weather report event. If the icon is selected the MPEG-4 weather report is launched.

The presentation of the mosaic screen is achieved by using an MPEG-4 scene description with preview trailers encoded in MPEG-4. With the use of MPEG-4, the user is able to interact with trailer objects on the screen, select menu items, and choose the position of additional information. Material for the mosaic interface of the trailer corresponds to the content created for the TV programs.

\section{B. Interactive Services}

MPEG-4 introduces the concept of scenes that are composed of many different, possibly arbitrarily shaped, video objects. The user can interact with each object in the scene, and the response will depend on which object is selected. This also allows accompanying meta-data and indexing information (MPEG-7) to be attached to single objects, not only to the scene as one entity.

In CustomTV, an interactive weather forecast application was implemented to demonstrate the aforementioned functionalities. The weather forecast for Europe is initially displayed with a map of Europe. Upon a user selecting a specific country or region, a more detailed forecast for the local regions is shown. These video objects can be zoomed in or out, and placed anywhere convenient in the scene by the viewer. He/she can also choose to listen to the audio object associated with the local forecast, or with the European one. In Fig. 3, the local weather forecast for Germany is selected and placed in the upper left corner of the European forecast.

\section{Data Services}

Enhanced services can include a variety of data services. These could be related to the current TV-programs or could be independent. Such information can be hidden or displayed, depending on the user's preferences. Such services can be efficiently encoded by using MPEG-4 scenes containing only text and graphics, and the most recent information can be made available through scene updates. CustomTV demonstrated two services as follows. 


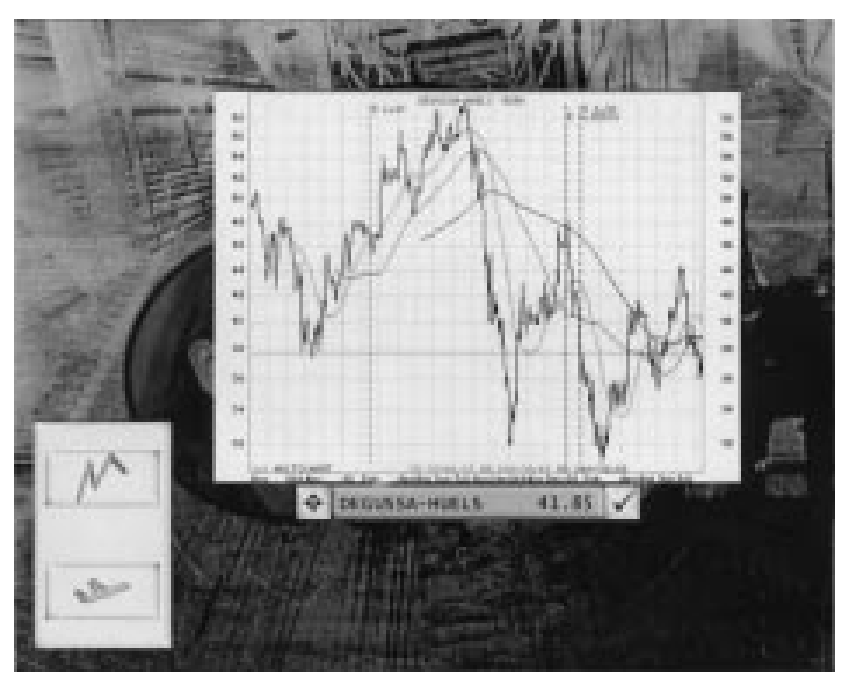

Fig. 4. Stock rate ticker scenario.

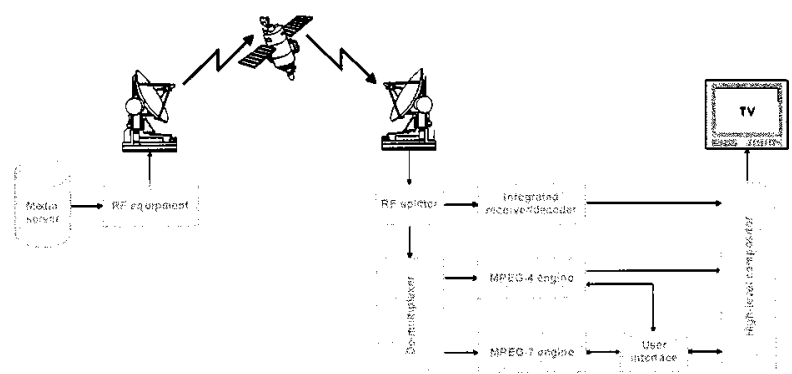

Fig. 5. CustomTV demonstration setup.

- Flight information: Flight information at a given airport can be accessed, such as departure and arrival times; each flight can be selected and tracked, e.g., to note when the plane has landed.

- Stock market: Information about individual share prices and their changes can be shown. One or more stocks can be selected and tracked, and graphs showing their variation over time can be placed anywhere on the screen.

Fig. 4 shows a typical screen-shot for these applications: an overview of the departure-times at an airport, tracking of two shares, and an ordinary television program in the background.

\section{Customized TV Demonstrator}

The demonstration setup had to be capable of showing MPEG-4 and MPEG-7 elements inserted in a DVB transport stream, as well as some convincing applications utilizing these elements. The demonstrator developed consisted of a real-time receiver system, which is fed from a media server in real-time via a satellite link (Fig. 5). There is no return channel from the receiver system toward the server side.

\section{A. Content Generation and Transmission}

MPEG-4 encoding of audio-visual material and multiplexing was performed mostly using the reference software available from the MPEG standardization consortium [5]. MPEG-4 audio/video content and Binary Information for Scenes (BIFS) MPEG-4 scene description data was also preproduced, multiplexed together using the intermediate Flex-Multiplexer and stored on a server file storage system. The BIFS description defined the position of MPEG-4 objects in the scene and created graphics objects. The MPEG-4 scenes themselves were programmed without help of any authoring tools, since such tools were not available at the time.

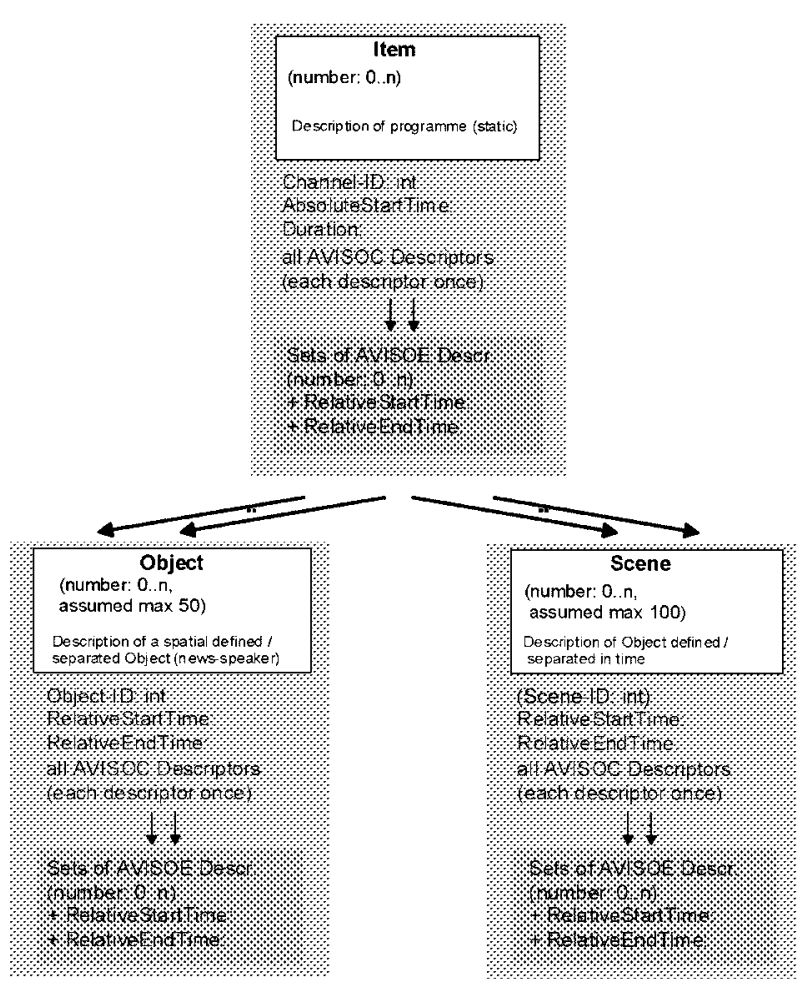

Fig. 6. Structure of the descriptors, which could be related to the video content.

MPEG-7 indexing information for all the MPEG-2 and MPEG-4 encoded content was provided by setting up an appropriate database. As the standardization of MPEG-7 had only just started (the complete standard will not be available before July 2001) the descriptors used were based on the project's early contributions to MPEG, and therefore may not be fully compliant with the final standard.

MPEG-7 indexing information that described associated MPEG-2 and MPEG-4 content was also pre-produced and stored on a server file storage system. All MPEG-4 and MPEG-7 information, as well as the MPEG-2 audio and video components, had to be included in one MPEG-2 transport stream. This was done using private sections, based on the work in the MPEG consortium regarding inclusion of MPEG-4 in MPEG-2 transport streams.

Enhanced DVB compliant Transport Streams carrying all the necessary MPEG-2, MPEG-4 and MPEG-7 data were created off line and stored on a media server. A real-time receiver system was built, and a satellite link provided to be able to play out the data from the server and transmit it to the receiver in real-time.

\section{B. BMPEG-7 Metadata Descriptors}

Special descriptors are events, which describe an action or event related to a time period like a goal in a soccer game. An overview of the structure of the metadata descriptors is given in Error! Reference source not found. AVISOC are audio/video content descriptors related either to a program, a scene or an object and AVISOE are audio/video event descriptors related either to a program, a scene or an object.

Since there were no encoding tools available, a simple scenario for manual (offline) generation of MPEG-7 like data was introduced. This data format is defined below. The stored MPEG-7 like data file was transmitted with the transmitted video content at a constant bit rate.

In the first processing step, the content of the static and dynamic descriptors is manually edited. This is done with the aid of Microsoft Excel, where templates guide and help the user to enter the data in an appropriate format. The use of a professional table-processing tool 


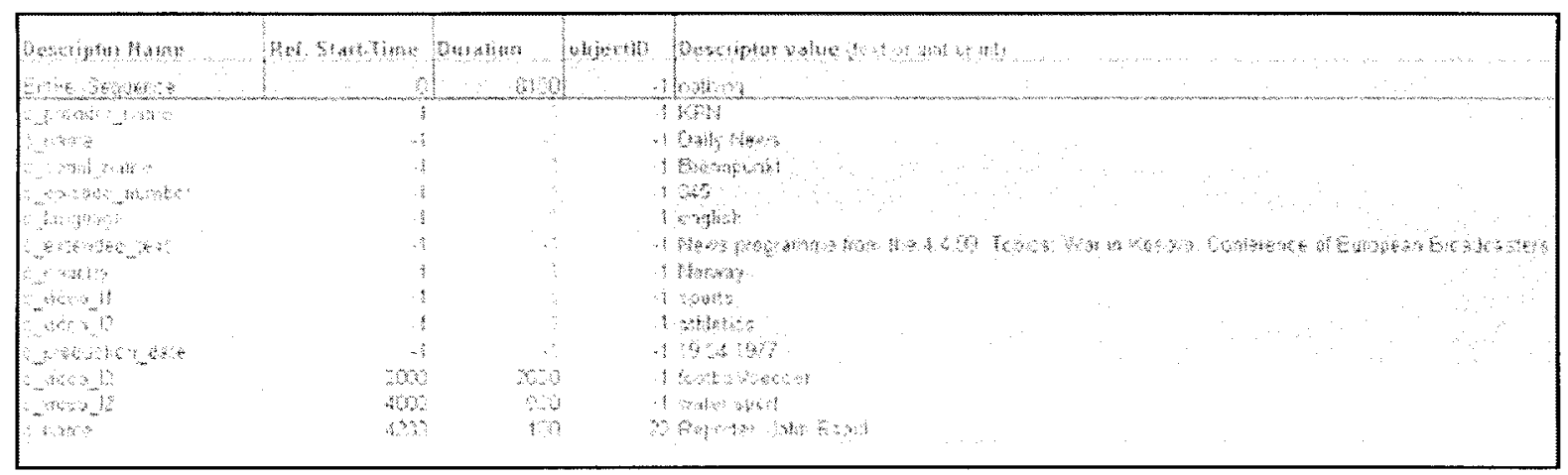

Fig. 7. Example of a completed Excel template for manual input of the descriptor values.

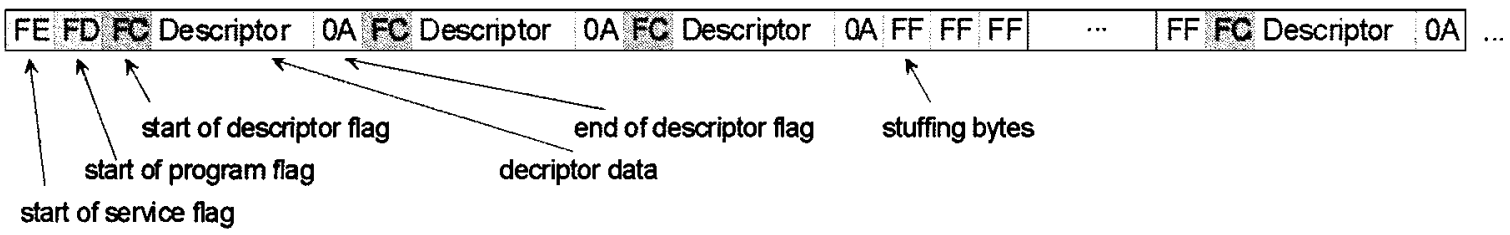

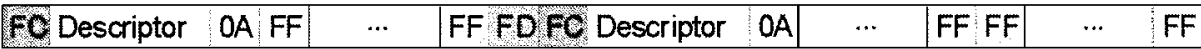

(a)

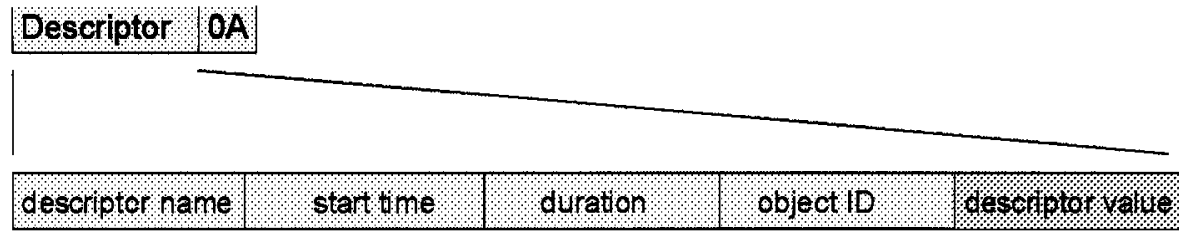

(b)

Fig. 8. MPEG-7 stream structure. (a) Description of the MPEG-7 like bit stream. (b) Subfields within the descriptor data field.

has the advantage of a convenient user interface and existing export filters. Also the data generated may be extended or modified easily by editing the stored data. Fig. 7 shows an example of the Excel template already filled in with the content for descriptors. An explanation of the different columns is given in the first line, and the second line is exclusively inserted to provide the total duration of the entire sequence in milliseconds. Each of the following lines is related to one specific descriptor.

The descriptors are divided into two categories, namely static and dynamic descriptors. Static descriptors are valid for the whole sequence whereas dynamic descriptors are only valid for a specific time period within the sequence. Within the template in Fig. 7 the timing information for static descriptors is set to the value -1 . All descriptors may be related to the whole program (sequence) or to one spatial object or to a specific scene. An object is a spatially separated element of an image like a speaker in the foreground, and a scene is a temporally separated period of a sequence. Descriptors, which are not related to a dedicated object, are marked through an objectID value of -1 .

In the second processing step a software module called the bit stream processor reads the data from the text file generated in the first processing step and prepares the bit stream file for multiplexing and transmission. The bit stream generator has to:

- provide additional information both to enable decoding and to allow resynchronization in case of packet loss during the transmission;
- produce a bit stream with an adjustable, but fixed, bit rate. It has to put the descriptors in the correct positions within the bit stream with respect to the relative start time of the corresponding descriptor.

Three kinds of flags, each of length 8 bits, are inserted as additional information for decoding and resynchronization. These flags indicate the start of a service (a concatenation of programs), the start of a program and the start of a descriptor.

The descriptor fields within the bit stream shown in Fig. 8(b) are divided into five subfields. The subfields contain the descriptor name, the start time (provided for prestored or pretransmitted data), the duration, the objectID, and the actual descriptor value. The five fields are separated by blanks.

The bit stream generator is able to process a variable number of textual input data, each related to one video sequence. By using the start of service flag, several sequences can be concatenated into services. The resulting MPEG-7 bit stream is thus related to the service (concatenation of several video sequences).

\section{Content Multiplexing}

The MPEG-4 and MPEG-7 data was inserted as MPEG-2 private sections in the Transport Stream, but without the PDAM-7 descriptors since at the time of implementation these were still not standardized. This meant that the Transport Streams generated still conformed to the 


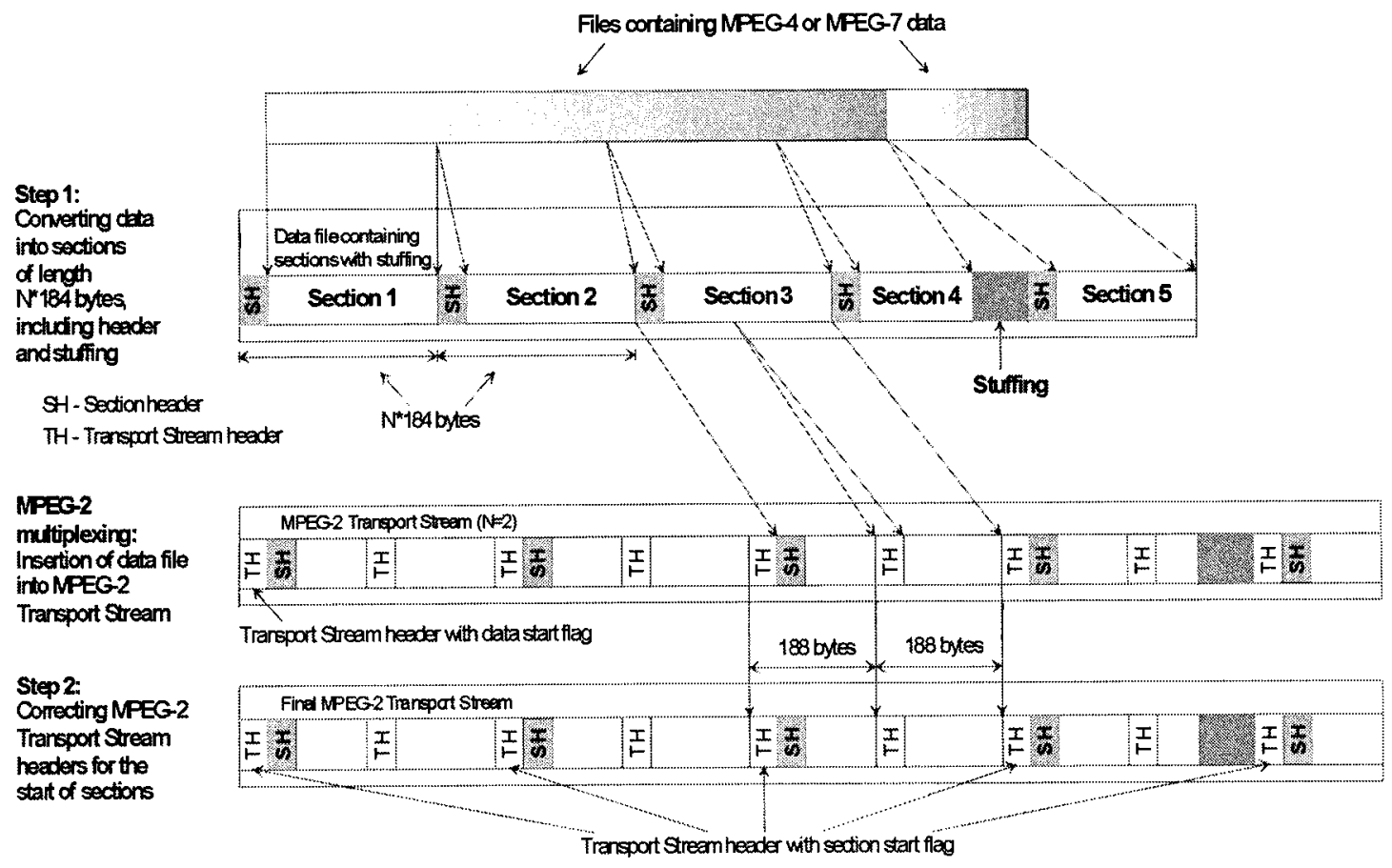

Fig. 9. Raw MPEG-4 and 7 data is converted into sections.

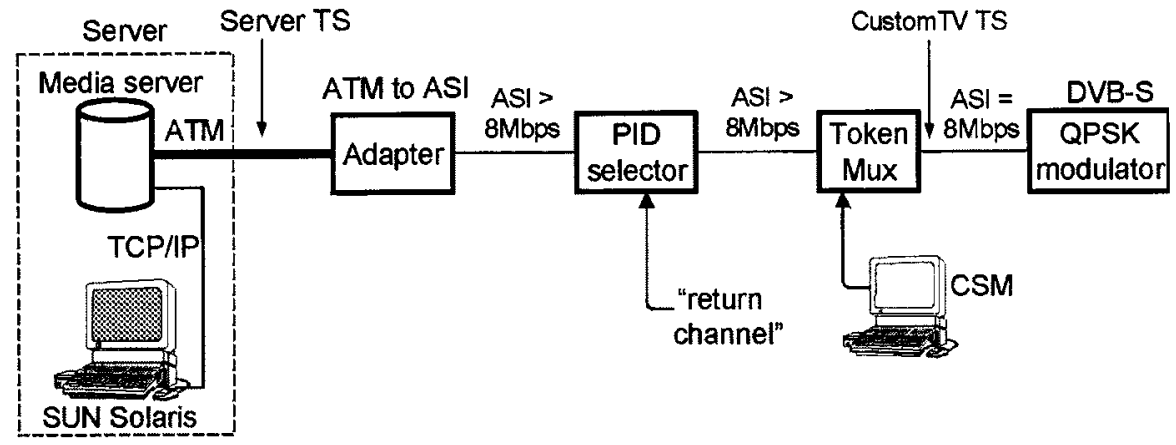

Fig. 10. Broadcast elements.

MPEG-2 standard (or DVB). Fig. 9 shows how raw MPEG-4 and -7 data files were converted into sections, which were inserted in a DVB MPEG-2 Transport Stream. The private section syntax is defined in the MPEG standard.

Since the content is created off-line, a server and broadcast chain was needed for storage and transmission, as depicted in Fig. 10. The elements were divided into three stages: the Media server and conversion of its output to DVB/ASI, PID editor, and Creation of the final multiplex and uplink.

The media server could play out several MPEG-2 Transport Streams at the same time, up to an aggregated maximum rate of $60 \mathrm{Mbps}$. Because of this, it is equipped with an ATM STM1, optical multimode (OC-3), 155 Mbps output.

The functionality of the PID editor was based on the fact that a TS consists of different elementary streams each with a different PID value. By changing the PID values that were not used in a given demonstration scenario to a stuffing PID number, it was possible for the TokenMux to let through the elementary streams needed in the MPEG-2 TS, without either reconfiguring the TokenMux or the receiver on the downlink side.

\section{RECEIVER SYSTEM AND USER INTERFACE}

The customized TV receiver system is shown in Fig. 11. This is based on a normal digital TV receiver which decodes the MPEG-2 stream received from the transport stream (TS). Our receiver, however, had a DeMux stage to extract the MPEG-4 and MPEG-7 associated streams to feed, respectively the MPEG-4 and MPEG-7 engines for decoding. The MPEG-7 scene was formed by a "Low level Compositor" which was then mixed with the TV sequence by a "High level Compositor." The MPEG-7 information, on the other hand, provided index information and events to a "Personal Agent" that processed this information. The User Interface provided the facility to set up a user profile and displayed information about programs, menus, prompts, etc. The video signal from the UI, together with the MPEG-2 and MPEG-4 audio and video signals were composited to the final image by the High Level Compositor using chroma keying.

The demultiplexing takes place on PID (Packet Identifier) level. All data inside a TransportStream is packetized into packets of 188 bytes where packets from the same stream (video, audio or private data) have 


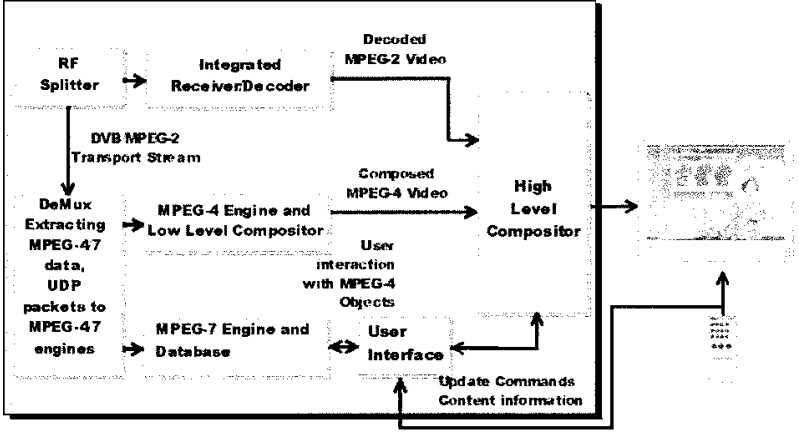

Fig. 11. Custom interactive TV receiver system.

the same PID number. Thus, selecting a component for extraction from a TransportStream is a matter of informing the demultiplexer of the corresponding PID number. It is not possible to extract the raw content of the 188 bytes Transport Stream packets. Sections were used for the transportation of the MPEG-4 and MPEG-7 data inserted in the Transport Stream, which is also compliant to the standardization within MPEG.

\section{A. MPEG-4 Engine}

The receiver incorporates an MPEG-4 decoding chain. It includes a set of important MPEG-4 tools.

1) MPEG-2/4 Extractor:

2) MPEG-4 DeMux:

3) Synchronization Layer (SL):

4) OD Decoder:

5) MPEG-4 Video Decoder:

The video decoder supports the MPEG-4 Main Profile without the scalable features and without grey-scale alpha. The C60 based hardware support makes the video engine capable of decoding up to 4 CIF sized videos at approximately 15-25 Hz.

6) MPEG-4 AAC Audio Decoder:

7) Low Level Compositor (LLC).

The BIFS decoder conformed to the MPEG-4 systems specification but only supported a 2-D subset of nodes. The BIFS decoder generates an internal representation of the hierarchical scene description that must be efficient for updating and rendering.

The renderer utilized OpenGL though it is only targeted for 2-D rendering. The following rendering and user interaction features were supported:

- Selection;

- Zoom and shrink by a factor of 2;

- Translation;

- Binary alpha planes;

- Depth sorting of objects.

The LLC received user interaction commands via the Java based interface from the UI/HLC. It provided a specific mode for the EPG scenario in which it displayed 4 out of $N$ trailers (e.g., still pictures) by splitting the display area into four equally sized tiles.

\section{B. BMPEG-7 Engine}

The MPEG-7 processing engine is the central for selecting channels, handling relevant events and extracting additional information as requested by the user.

Fig. 12 shows a schematic diagram of the MPEG-7 engine with two external inputs. The Central part of the MPEG-7 engine is memory contains relevant information delivered by the MPEG-7 decoder. This information is intended to be suitable for event processing, matching and information extraction. Not all information for all the available channels that is provided by the MPEG-7 stream needs to be stored in memory. Some information is updated continuously, such as event descriptions or categorizing information, and some is extracted from the MPEG-7 data on demand, such as additional information for the channel displayed currently. The specification of the data to be stored is done by a control unit, which processes the requests and interacts with the user interface.

The MPEG-7 engine is designed to perform the following functions:

- Receive the MPEG-7 data stream from the multiplexer and decode the data stream.

- Provide a list of channels that fit a requested category, such as sports, news, weather forecast, documentation, etc. In a first step the list of categories is fixed, consisting of a subset of the category list in the DVB-SI standard.

- Handle incoming events. After the user interface informs the MPEG-7 engine of the kind of events are of interest, the events have to be detected and the type of event and possibly a textual description of the event is delivered. In the first step, the event types should be compliant to the fixed event type list.

- Deliver additional textual descriptions that may be included in the MPEG-7 data stream for a selected channel or for an object within the scene of a selected channel.

- Provide a list of channels if a certain textual descriptor fits a given string such as the name of an actor or the type of an object within a scene.

\section{USER INTERFACE}

The CustomTV user interface is designed with the aim of integrating the enhanced services made possible by MPEG-4 and MPEG-7 as a natural extension of normal residential TV viewing. This is pursued, first, through the adoption of a layered approach to the introduction of more complex services. The default viewing mode takes the form of a conventional broadcast and the additional CustomTV services, accessed via an optional navigation bar, layered on top. These extra services, including the navigation bar itself, are displayed only on request. A second design constraint is that all user interaction is via a remote control that only provides a pointer (i.e., no keyboard input) and is based on a simple object-action style of interaction. The user interface is also designed to engage, as far as possible, with the user's conceptualizations of the type of content or service provided and independently of the specific technology or protocols used to implement it. For example, the user interface does not respect the distinction between playback of MPEG 2 or MPEG 4 video at the level of user interaction. From the user's point of view both types of video are treated as programs and the same basic actions are associated with them. In addition to combining technically distinct types of content, UI distinctions were also introduced between types of content delivered in a single format. For example, data services, trailers and programs are all supported by MPEG 4 but, from the point of view of the user, are treated as distinct types of service, each with a different interaction model.

The principal conceptual difference between user interaction with conventional broadcast television and the customized TV user interface lies in the choice of basic level objects for the interaction. While conventional television viewing is organized around channels and, perhaps, content provider, the customized TV user interface builds on individual programs as the basic-level objects around which user interactions are organized. As a result, user actions and navigation operate by default on the current program being viewed or, in the case of the electronic program guide (EPG), the trailer currently selected. This contrasts with the channel-based organization of conventional television and has significant consequences for navigation and interaction. For example, in CustomTV the "back" button operates in a manner analogous to a web browser and moves the user to the most recent program viewed (where available). In 


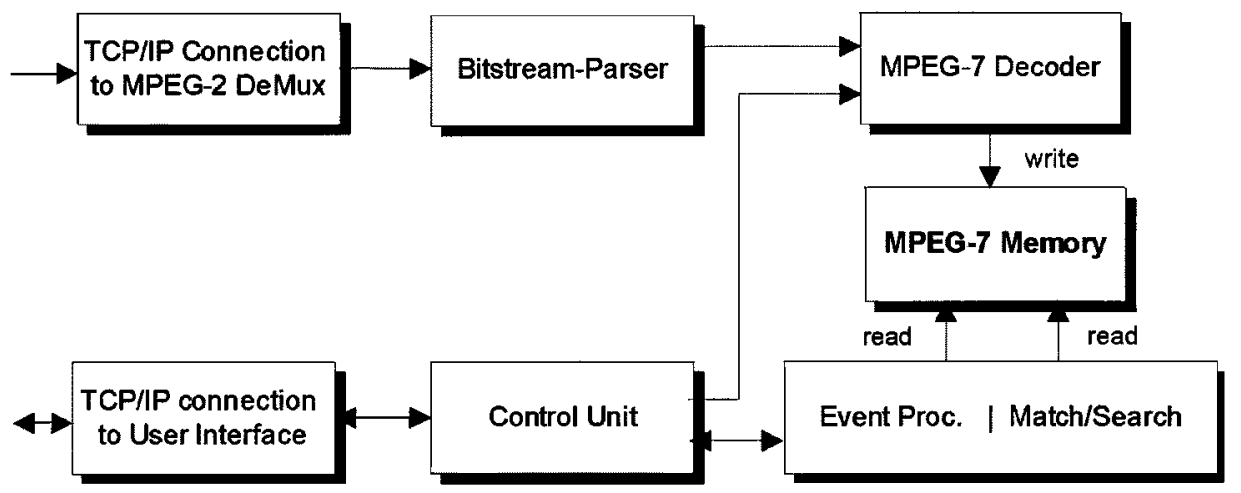

Fig. 12. Schematic diagram of the MPEG-7 engine.

conventional television, navigation is typically between the numerically ordered channels. In addition, navigation with the "up" and "down" buttons in CustomTV is structured according to user-preference information set in user profiles. These buttons move the user up and down a preference hierarchy rather than between channels. This organizing principle extends to the CustomTV EPG, where the order of presentation of trailers for currently available programs is determined by preference ratings for program categories. The more strongly preferred categories of program are placed nearer to the top. We propose that this represents a more natural and user-centered approach to viewing than is provided by interactions that are organized in terms of channels or content providers.

The user interface is completely implemented in Java. It consists of three main visual parts: the EPG, the Profile Editor, and the Video/ Interactive services. Objects representing the MPEG-4 and MPEG-7 engines, the IRD and HLC provide a clean programming interface and abstract these devices/modules away from the underlying control communication. The program manager maintains a list of all the current programs, ranked according to the user's interest specified in the active user profile. When a new program starts the MPEG-7 object notifies the user interface. It then creates an event to update the visual part of the EPG, and if the new program is more interesting than the one the user is currently watching, it creates another event that informs the user. The profile editor allows the user to indicate his preferences by giving a weight to items of interest that have been arranged in a tree structure (described previously). The video/interactive services component displays the normal MPEG-2 video and the interactive services. The selection of the right channel is managed by the IRD object. However, the implementation selects PID ranges by controlling the editor, and does not control the IRD itself. The event manager handles all events, both coming from the MPEG-7 Engine and internal ones (e.g., a more interesting program has started).

\section{EVALUATION}

To evaluate the demonstration system ten usability walkthroughs were conducted, using the live system and using a video recording [6]. Although this number of subjects is too small for reliable statistical generalizations the aim here was to carry out a formative evaluation designed to detect major usability issues [7]. Five people with significant expertise in advanced TV applications and five nonexpert participants were sampled.

Three scenarios were used which involved asking the user to carry out generic tasks, such as using events, program information, and preference features and also more specific tasks related to the EPG, Data Services (Stock Rates and Flight information), and the Weather Forecast Service. Overall, the evaluation of the user interface and the CustomTV services was positive. Participants were able to complete all the tasks presented to them. Both ease of interaction with the user interface and the usefulness of the functionality offered by the novel features were consistently rated as above average. General comments indicated that participants perceived the additional interactive features provide by CustomTV to be valuable extensions to conventional viewing. The positive perception of participants to customized TV was primarily attributable to the additional control afforded by specification of preferences and interest in particular events. The event "alert" or messaging feature was rated as particularly useful because it enabled the user to switch quickly between programs of interest. The program information feature and its contents were also perceived to be of high value. The most significant usability problem directly identified with the CustomTV user interface was the remote control. The $33 \%$ of participants found the button-based mouse controller very hard to use. Inconsistency in the icons used across the interfaces to different system features was also identified as a minor usability problem. This was particularly an issue where there were clashes between the basic user interface icons and those associated with data services. A more nebulous and perhaps more interesting problem for CustomTV and similar, preference based, models is that, in effect, they presuppose a single-user model of television viewing. This was not encountered as a problem in the evaluations since they were also restricted to single-user scenarios of use. However, much residential viewing is a social activity often involving groups of people such as friends and family [8]. In this context, the value of a preference based organization of, for example, the advanced EPG is undermined since people's preferences vary. Digital technologies provide the possibility of more flexible forms of television viewing and CustomTV demonstrates how this flexibility can be used to support sophisticated forms of personalization and customization. However, these technologies have not yet been applied to providing effective support for the social aspects of television viewing.

\section{CONCLUSION}

The CustomTV project has shown the potential of the MPEG-4 and MPEG-7 standards in introducing customized value added services by integration of advanced multimedia-content (based on MPEG-4) and indexing (based on MPEG-7) to conventional digital broadcast transport streams (MPEG-2). Special attention has been devoted to demonstrate the possible impact of the emergent MPEG standards to lead to open systems, in particular for set top boxes, to create novel services for traditional broadcasters. We have addressed typical challenges of digital television, such as the need for customized navigation and filtering mechanisms for the significantly increased number of programs in the digital domain. We have shown how the need for describing the interactive behavior of single objects on the screen can be met in $a$ standardized way. Typical examples for these objects are segments of a picture, but we have also demonstrated that this view can be extended to overlay graphics and switch buttons. 
CustomTV was followed by another European Union project called SAMBITS which extended many of the ideas developed to include a return channel via the Internet and combine Internet functionality to many of the services proposed. At that stage, the DVB MHP (Multimedia Home Platform) standard, not available for CustomTV, was already advanced enough to be adopted by the new project. MHP is expected to become a standard for Set-Top-Boxes which will make the potential use of standards for broadcasters even more immediate than the time when CustomTV was demonstrated.

To take a wider view, many of the standards mentioned here are already in use in the Internet community or very likely to be used in the future. Thus, the approach adopted by the CustomTV project it intended to show that the convergence of broadcast and Internet media can be seen as a distinct reality thanks to the harmonization of the standards used. Common and open standards, however, will give a significant push for further penetration of digital television broadcasting, especially in those countries where just the increased number of programs by itself does not create enough added-value to convince users "to go digital."

\section{REFERENCES}

[1] J.-P. Evian, “The multimedia home platform-An overview," EBU Tech. Rev., no. 275, pp. 4-10, 1998.

[2] S. Heimbecher, "First outing for MHP at IFA 99," EBU Tech. Rev., no. 282, p. $5,2000$.

[3] R. Koenen, "MPEG-4 multimedia for our time," IEEE Spectrum, vol. 36, pp. 26-33, 1999.

[4] F. Nack and A. T. Lindsay, "Everything you wanted to know about MPEG-7, Part I and Part II," IEEE Multimedia, vol. 6, no. 3 and 4, 1999.

[5] J. P. Cosmas, Y. Paker, A. Pearmain, and P. Schoonjans et al., "CustomTV system \& demonstration," in Proc. IEEE Int. Conf. Multimedia Computing and Systems (ICMCS'99), vol. 2, Florence, Italy, 1999, pp. $1110-1113$.

[6] P. Healey, A. Vaduva, P. Schoonjans, Y. Paker, and A. Pearmain, "User interface design for CustomTV," in Int. Conf. Media Futures, Florence, 2001.

[7] J. Nielsen, "Estimating the number of subjects needed for thinking aloud test," Int. J. Human-Computer Studies, vol. 41, no. 3, pp. 385-397, 1994.

[8] D. Morley, Family Television: Culture, Power and Domestic Pleasure. London: Comedia, 1986.

\section{An Amplifier Linearization Method Based on a Quadrature Balanced Structure}

\author{
Abelardo (Belex) Podcameni and Carlos Renato Riback
}

\begin{abstract}
A quadrature balanced structure is presented for linearizing amplifiers. It works by reducing the most annoying distortion component: the third-order intermodulation. This arrangement has some advantages over the negative feedback method, which is prone to oscillations. It also has advantages over predistortion and feedforward linearization methods, as it is simpler and needs no adjustments. Theoretically, third-order intermodulation products would be totally eliminated when using the proposed arrangement. In practice, it was observed that, even with nonperfectly balanced amplifiers, the third-order intermodulation level was reduced up to $17 \mathrm{~dB}$. The fifth-order intermodulation components were not reduced.
\end{abstract}

Index Terms-Amplifier linearization, feedforward, interception point, negative feedback, predistortion, third-order intermodulation.

\section{INTRODUCTION}

Broadcasting deals with transmitting radio waves to several users. Usually, the effective irradiated power level is at a paramount. One of the most stringent broadcast limitations resides on the nonlinearity of the final transmitter stage. If the transmitter was meant to irradiate a single continuous wave $(\mathrm{CW})$, the amplifier nonlinearity would only produce integer multiples of the working frequency-harmonics-which would be extremely easy to be filtered. In practical cases, the transmitter works with a multifrequency signal. Some of the nonlinear products will occur within the useful band. These undesirable signals, or intermodulation products, can not be eliminated by simple filtering action. Additionally, at the reception side, they will be perceived as noise. The situation is such that, in most cases, the intermodulation level establishes the ultimate working power of a transmitter. For a given task and specification, if one moves from a nonlinear to a linearized situation, a sensible higher power level may be obtained.

Hence, amplifier linearization is an activity that has historically deserved quite a lot of attention, with the Telecommunications field benefiting from that activity. Techniques like negative feedback [1], predistortion [2]-[, ] and feedforward [3] have been described and implemented. Each one of such techniques presents advantages and disadvantages. With negative feedback, the phase-shift around the loop must be tightly controlled in order to avoid oscillations. Predistortion and feedforward tend to have their efficiency depend upon multiple adjustments, jeopardizing mass production.

A linearization scheme, based on a quadrature-balanced structure is described here that needs no adjustments. Additionally, it is a very stable one, as it does not possess a feedback loop.

Section II describes the proposed arrangement and carries out the demonstration that the third-order intermodulation product would theoretically be eliminated. Section III presents the experimental part of the work, considering first a nonlinearized amplifier and then a similar linearized amplifier. Section IV discusses the results.

Manuscript received January 3, 2002; revised March 11, 2002.

A. Podcameni is with the Telecommunications Studies Center of the Catholic University of Rio de Janeiro, GSOM/CETUC/PUC-Rio, 22453-900, Rio de Janeiro, Brazil (e-mail: abelardo@ cetuc.puc-rio.br).

C. R. Riback was with the Brazilian Navy. He is now with the Telecommunications Studies Center of the Catholic University of Rio de Janeiro, GSOM/CETUC/PUC-Rio, 22453-900, Rio de Janeiro, Brazil.

Publisher Item Identifier S 0018-9316(02)05258-7. 